\title{
LA VITA OPEROSA DI MASSIMO BONTEMPELLI FRA NARRATIVA, AUTOBIOGRAFIA E «AUTOFICTION»
}

\author{
Cinzia GALlO \\ Università di Catania
}

\begin{abstract}
En): From the earliest pages, Massimo Bontempelli's La Vita operosa (1921) seems to mix the characters of an autobiography, a narrative text and, it could be said, of an autofiction. The first is certainly the re-enactment by the narrator of some facts of his own existence, the reminder attributed to the memories, the autobiographical pact, justified by the subtitle (Avventure del '19 a Milano), established with the readers, often called in question. Various situations and characters, however, are clearly unlikely: the Daimon with whom he maintains constant dialogue, the golden "waterfalls" pouring into the streets, the magical mirror with which the seventh chapter ends. All this, in line with the program of magical realism, would seem to authorize the recall of some aspects of Doubrovsky's autofiction. On the other hand, the individual "adventures" of the narrator, who does not hesitate to express controversial judgments about the society around him, are real selfcontained narrative inserts, as shown in the subdivisions of the chapters. So, in the text, we have three planes intersecting. And this makes Bontempelli's operation more important, an operation that does not run out, therefore, only in the will to rebuild space and time.
\end{abstract}

Keywords (En): autobiography ; fiction ; autofiction ; modernism ; magical realism

Parole chiave (It): autobiografia; narrativa; autofiction; modernismo; realismo magico

\section{Introduzione}

«La Vita intensa e la Vita operosa costituiscono due delle esperienze più originali del primo Bontempelli [...]» afferma Marinella Mascia Galateria (1977: 186). I due testi, in effetti, sono stati sempre accostati dalla critica che, comunque, ha riconosciuto nella Vita operosa una «delle più felici "allegorie" critiche del primo Novecento» (BALDACCI, 1959: 438), «il momento in cui era cominciata la riflessione» (GIORDANO, 2009: 18) verso la fase del realismo magico, «la prefigurazione del tema dell'alienazione» (FonTANELlA, 1997: 25). Appunto perché testo più maturo rispetto alla Vita intensa, e fase di snodo nella poetica di Bontempelli, è opportuno partire da questo testo per analizzare un aspetto abbastanza costante nella produzione del nostro autore, il particolare rapporto che si instaura fra gli elementi narrativi e i diversi statuti dell'io. Difatti, «L'io narrante bontempelliano non è solo colui che racconta ma è anche colui che si pone come personaggio stesso dei racconti narrati, partecipando direttamente agli avvenimenti e divenendone il protagonista principale» (GIORDANO, 2009: 29), oltre a presentarsi, talvolta, come autore ( $«$ Non ignoro che se queste pagine saranno lette da qualche curioso tra molti anni, forse egli trarrà da alcune delle mie parole ragioni d'incertezza e di dubbio» $\left.{ }^{1}(\mathrm{VO}, 226)\right)$.

\footnotetext{
${ }^{1}$ La sigla VO indica La vita operosa (BonTEMPELLI, 1978).
} 


\section{Finzioni dell'io}

\section{- L'io personaggio autobiografico}

Fin dalla prima riga, la Vita operosa sembra avere le caratteristiche di un' autobiografia. Vi rimandano, sicuramente, il rilievo attribuito ai ricordi, sottolineato dall'alternanza, quanto all'uso dei verbi, fra presente e passato; ${ }^{2}$ il patto autobiografico, giustificato dal sottotitolo (Avventure del '19 a Milano), instaurato con i lettori, spesso chiamati in causa, secondo il procedimento della metalessi $;{ }^{3}$ alcuni commenti del narratore; ${ }^{4}$ la rievocazione, da parte dell'io narrante, di alcuni fatti della propria esistenza. In alcuni casi essi coincidono con la vita autentica di Bontempelli. Sappiamo, infatti, che l'io personaggio, dopo aver partecipato alla prima guerra mondiale, rientra a Milano, dove vive la condizione di precarietà che anche Bontempelli conosce e che sembra contraddistinguere, come evidenziato già nella Vita intensa, l'attività di scrittore. Se da una parte, difatti, i nuovi tempi non sono a questa favorevoli, dall'altra l'io personaggio sottolinea, con un'ironia che scaturisce oggettivamente dai fatti, l'esiguo valore della letteratura contemporanea: «Aspettando in Galleria, mi lessi con gran cura tutti i titoli dei libri nelle vetrine di Treves e di Baldini e Castoldi; con la quale esplorazione mi misi in breve e compiutamente a giorno degli spiriti e delle forme della nostra letteratura contemporanea» (VO, 166); «"in affari" ammonisce "non ci sono generi". "Come in letteratura?"» (VO, 209). È palese, a questo proposito, il collegamento alle riserve espresse da Bontempelli. Ne L'avventura novecentista, egli afferma:

Il panorama della letteratura nostra contemporanea io lo vedo [...] nel modo seguente: / Da una parte c'è un certo numero di lavapiatti, dall'altra un certo numero di cacastecchi. Molto numerosi l'uno e l'altro gruppo, e la gente dall'una e dall'altra parte sta pigiata. In mezzo uno spazio ampio, in cui si muovono, con molta libertà e senza darsi l'un l'altro alcun fastidio, pochi valentuomini, cioè gli scrittori autentici. (BONTEMPELLI, 1974: 147)

Pure alcuni aspetti della personalità dell'io protagonista coincidono con quelli di Bontempelli. L'io personaggio esprime decisamente le proprie opinioni («Io

\footnotetext{
2 «Ricordo che il quaderno [...] era fatto a domande e risposte [...]» (VO, 149); «Ricordo che parecchi anni sono [...] solevo entrare in quel caffè [...]» (VO, 153); «Ignoro se le persone che vi si trovavano rappresentassero ancora il fiore dell'intellettualità cittadina» (VO, 154); «uno sbadiglio quale non ricordo d'aver messo insieme il simile mai nella mia vita» (VO, 207); «Ricordo quell'istante della mia vita come un turbine [...]. Non ricordo come e a chi pagassi il doveroso contributo [...]» $(\mathrm{VO}, 242)$; «Andandomene a piedi (non ricordo il perché, [...]) verso il bar del Commercio» (VO, 264).

${ }^{3}$ È sufficiente qualche esempio: «Ma a quel punto io sentivo un vuoto improvviso. Forse qualcuno dei lettori l'ha sentito con me. M'auguro siano pochi: li avverto che è un fenomeno morboso, [...]» (VO, 150); «Nessuno dei lettori odierni può confondere il tricolore ufficiale con quello politico di cui ho parlato» (VO, 226).

4 «ciò non ebbe alcuna importanza nella mia vita come non ne ha alcuna nel séguito di questo racconto» (VO, 179); «Un invincibile spirito di avventura ha sempre spinto la mia vita ad accogliere di buona voglia ogni invito dell'imprevisto» (VO, 229); «quel mio sforzo, [...] non si sarebbe inserito in alcun modo nella serie della mia biografia, [...]» (VO, 277); «Basti dire che è uno degli innumerevoli amici avvalangatimi dalle multiformi vicende della mia vita» (VO, 279).
} 
invece non ho mai evitato di compromettermi. Ho sempre ignorato la virtù della prudenza. Mio danno, e mia passione» (VO, 159)), allude al proprio passaggio da una formazione classica al giornalismo e alle proprie simpatie per le arti figurative

Forse un tempo, quand'ero immerso in classici studi, avrei pensato di scrivere su Belloveso una truculenta e compassata tragedia. Più tardi, poi che la vagante sorte m'ebbe sfiorato con le ibride penne del giornalismo [...] avrei tentato di quietare lo spirito di Belloveso e il mio con una serie di articoli agitanti la proposta di un monumento: tutti gli scultori e i procacciatori di Comitati sarebbero stati con me.» (VO, 199)

Fa riferimento a propri componimenti: «Non ci vado neppure, per ragioni profonde che ho spiegate in un mio racconto intitolato Florestano e le chiavi che mi permetto di citare $[\ldots] \gg(\mathrm{VO}, 232)$.

A rafforzare i caratteri dell'autobiografia contribuiscono, pure, le precise indicazioni cronologiche: «in un giorno di gennaio del 1919» (VO, 165); «un anno avanti lo scoppio della guerra europea» (VO, 177); «fu nel febbraio del primo anno del dopoguerra» (VO, 225); «E da quel giorno a oggi che scrivo son passati altri quindici mesi che è a dire circa cinquecento giorni cioè quasi mille pasti» (VO, 235).

\section{- L'io personaggio fittizio}

Questi stessi meccanismi, usati in funzione ironica, ${ }^{5}$ sono la spia del carattere fittizio dell'autobiografia. L'io narrato, cioè, si trova invischiato in situazioni ed episodi palesemente falsi, che rimandano, da una parte, al modernismo, dall'altra al realismo magico. Egli, innanzitutto, appare disorientato, privo di punti di riferimento, incapace di inserirsi nella città di Milano che, dopo la prima guerra mondiale, ha cambiato il suo volto rispetto al passato: «produrre» è adesso più importante che «inventare» (VO, 168). Ricorrenti sono perciò i termini «disagio» (VO, 205, 221, 269), «turbamento» (VO, 232), «inerzia» (VO, 243), «precarietà» (VO, 239).

L'io narrato vive, dunque, la stessa condizione di disorientamento, già evidenziata nella Vita intensa, ${ }^{6}$ dell'eroe modernista $^{7}$, come attestano alcune metafore:

\footnotetext{
5 «Quale solenne, invidiato ed esemplare collocamento nel mondo sociale avrei io oggi, se quella mattina, un anno e un terzo fa, la mattina del 22 di febbraio del 1919, primo anno di quel Dopoguerra, se quella mattina io fossi andato da Sua Eccellenza» (VO, 277). Ad un memoriale, poi, si fa riferimento nella fabula: «Stesi questo piano in un accurato memoriale. [...] Compilato il mio complesso memoriale artistico-storico-finanziario-politico, lo portai a copiare in dodici esemplari a una dattilografa fidata: [...]. / Dopo avere riletto e corretto il memoriale m'indugiai per poco in qualche pensiero domestico» (VO, 202-203); «Il giorno 9 febbraio del 1919, alle ore 10 e 45 del mattino, mi venne il mal di capo» (VO, 247).

${ }^{6}$ L'io protagonista, per esempio, per due volte, trovatosi sul tram, non scende alla fermata giusta. Prova, quindi, un «inesplicabile disagio» di fronte all'improvviso benessere, successivo ai suoi «tre anni di vita randagia» (BONTEMPELLI, 1978: 90).

7 Già Luperini inserisce Bontempelli nel modernismo (2012:11) e Patrizia Farinelli parla del novecentismo come di un' «isola [...] nell'arcipelago modernista» (2016:5).
} 
[...] mi sono ritrovato nell'aperta campagna Milano, senza bussola, né orologio, né sole, né stelle. / Ho girato dunque per la città, respirando la vita e cercando affannosamente un albero per vedere da che parte sta il nord» $(\mathrm{VO}, 151)$;

$\mathrm{Ci}$ sono dunque tornato quel giorno che mi aggiravo alla ricerca d'un albero orientatore. (VO, 154);

Io mi sentivo più che mai senza bussola. (VO, 155);

Nella cattedrale del dio Oggi non sono punti cardinali. Non è necessario orientarsi. Basta la conclusione dei più intelligenti tra i miei compagni della scuola di Artiglieria: «Ognuno farà quello che potrà.» $(\mathrm{VO}, 159)$

L'io narrato si dimostra così incapace di gestire la propria vita quotidiana (è definito, del resto, «buono a nulla» (VO, 191)): manifesta la propria «insufficienza dell'io» ${ }^{8}$ licenziandosi dall' agenzia di pubblicità presso cui era stato assunto, fallendo nel suo incontro con il «pescecane» (VO, 188) e nel tentativo di portare a termine la via Belloveso e, alla fine, rinunciando al colloquio con un importante ministro. Questo atteggiamento lo pone, però, nella condizione di poter osservare e criticare la società contemporanea, volta essenzialmente al guadagno materiale, all'apparenza, mentre i tradizionali valori della convivenza civile, della letteratura, della giustizia passano in secondo piano. Bontempelli utilizza, a questo scopo, il meccanismo dell'antifrasi (alla vacuità della società corrispondono i caratteri cubitali della parola 'oggi', presentata in cartelloni pubblicitari), il linguaggio religioso in funzione parodica. Le vicende dell'io narrato si uniscono talvolta alle considerazioni dell'io narrante: «Il Verbo è eterno, ma le sue incarnazioni sono caduche come gli assi delle impalcature, [...] Ma a tutti gli dèi [...] a Cristo stesso, succede ora, [...] il nuovo Dio, che si chiama / OGGI» (VO, 157); «Nella cattedrale del dio Oggi non sono punti cardinali» (VO, 159). L'avverbio 'Oggi', perciò, inserito «in un contesto lessicale paramitologico religioso [...] mette in moto quello che sarà il meccanismo sotterraneo [...] dell'intero testo, cioè la denuncia dei falsi miti collettivi, ma anche l'esorcizzazione delle reali, inquiete dimensioni dell'incipiente "operosa" civiltà di massa» (SACCONE, 1979: 19).

L'io narrato, quindi, paragona il direttore dell'agenzia ad una statua, a significare come la pubblicità, concepita come una vendita di idee, svuoti di consistenza gli esseri umani: «ei [Gattoni] s'era ritto in piedi e teneva la destra poderosamente infilata nell'apertura del panciotto. Così stette un istante, fissandomi, immobile come la statua di Bartolo che gli faceva da sfondo» (VO, 169). Il linguaggio del corpo, verso cui Bontempelli mostra sempre una certa propensione (pensiamo alla dialettica animato / inanimato che caratterizza Miracoli o Eva ultima), attesta invece come la società metta in primo piano l'apparenza, l'esteriorità:

Cercavo, con lo sguardo abbagliato, la barba alla Palmerston d'una persona qualunque; invece mi venne incontro un personaggio importante, adorno di un'elegante e contenuta pinguedine, e tutto raso: una faccia quadrata, un mento quadrato; anche la testa era quadrata $[\ldots]$. (VO, 167)

\footnotetext{
${ }^{8}$ Donnarumma, 2006: 25.
} 
L'epifora rafforza la connotazione negativa dell'aggettivo.

Altre volte, l'io narrato si serve di paragoni e metafore, in linea con la tendenza, espressa da Bontempelli in «900» e poi ne L'avventura novecentista, a stabilire relazioni fra letteratura ed arti figurative:

Mi trovavo dunque nel centro di quella terza Babele su cui Valacarda aveva predetto prossimo non so che fuoco divino o sociale. [...] Le immagini mi presero nel loro possesso. L'aria della sala intorno a noi si fece liquida e verde come in fondo a un oceano, oceano scivolato da grandi belve corsare che aprivano gole di Satana, arrotavano i denti alle rocce subacquee, e come d'intesa movevano tutte obliquamente, leviatani torvi, dall'abisso gelido in su, verso un'alta luce d'incendio lontano che le traeva; e il corteo non aveva fine, sempre più enormi e nere, con la smorfia d'un ringhio di cui non s'udiva la voce, battendo le code nel liquido muto, su, verso l'alto. (VO, 190)

Affermando di scrivere «per i posteri» (VO, 188), così come era avvenuto ne La vita intensa, ${ }^{9}$ l'io narrato sottolinea, inoltre, come tutti gli intellettuali si trovino esclusi dalla società, incapaci di comprenderne i meccanismi. Lo afferma Gattoni, servendosi dell'interrogativa retorica, delle enumerazioni: «[...] non sente come tutto questo puzza di letteratura? Di scuola e di letteratura, professorume e scrivaneria» $(\mathrm{VO}, 174)$.

Bontempelli riprendeva, in tal modo, quanto asserito da Pirandello alla fine de La tragedia di un personaggio: «Ella va cercando, oggi, tra noi, uno scrittore che la consacri all'immortalità? Ma guardi a ciò che dicono di noi poveri scrittorelli contemporanei tutti i critici più ragguardevoli» (1937: 703).

D'altra parte, l'esistenza è, secondo i principi del modernismo, dominata dal caso, «la vagante sorte» (VO, 199):

Io ho sempre pronte diverse città natali a seconda delle varie occorrenze della vita. (VO, 197);

Ma il caso m'impigliò ivi in una complicata e mirabile avventura [...] (VO, 248);

oggi è necessario imparare a vivere giorno per giorno. (VO, 254);

paese [...] appariva abbandonato a se stesso e alla malfida signoria del dio Caso. (VO, 226)

Non esistono, dunque, certezze, tanto che perfino le favole e i miti, che poi, invece, corrisponderanno «"ad un tipo di narrativa collettiva ed anonima" ${ }^{10}$ (FARINELLI, 2012: 25), risultano essere «scarsamente probanti» (VO, 162).

Ricordando una leggenda poco nota su Ercole, il Dàimone, con cui il protagonista dialoga costantemente, in una sorta di scissione della sua personalità, «tra l'uomo comune e l'uomo filosofo» (VO, 278), mette in discussione i tradizionali concetti di bene e di male, in linea con un relativismo di impianto modernista:

\footnotetext{
9 «Lo scrivo per i posteri», leggiamo ad apertura de La vita intensa (BONTEMPELLI, 1978: 7). Qui, poi, grande rilievo ha «L'appuntamento» (BONTEMPELLI, 1978: 18), richiamato pure ne La vita operosa: «Avevo un appuntamento d'affari, [...]» (VO, 229); «aveva dato con brevi parole un misterioso appuntamento» (VO, 167)..

${ }^{10}$ Molto spesso Bontempelli usa il mito e la tradizione letteraria in senso parodico.
} 
La leggenda poco nota è questa: Ercole fin da ragazzo aveva sentito dire molte volte da Alcmena che la virtù è bellissima e il vizio orribile. Trovatosi al bivio, vedendo una strada brutta e fetida si cacciò subito in quella, convinto di entrare nella strada del vizio. Quando s'accorse dell'errore non era più a tempo a tornare indietro; ciò che del resto è avvenuto e avviene in ogni tempo anche a uomini comuni, [...]. (VO, 163)

Egli, poi, privo di consistenza, ad un certo momento si materializza, sottolineando come l'aspetto esteriore, il corpo, sia solo una forma priva di reale valore: «Io non ho nessuna forma: dovrò copiarne una qualunque, la prima che mi venga in mente» (VO, 270). Criticando, comunque, il comportamento di tutti coloro che «ignorano la vita dello spirito. [...] correndo alla soddisfazione momentanea degli appetiti più bassi» (VO, 275), si precisa quale alter ego di Bontempelli, per cui asserisce che «il tempo e lo spazio sono infiniti ${ }^{11}$ (VO, 275).

Non c'è dubbio, d'altra parte, che la figura del Dàimone rimandi al realismo magico, secondo il noto programma esposto in «900» e poi ne L'avventura novecentista: «Il mondo immaginario si verserà a fecondare e arricchire il mondo reale» (BONTEMPELLI, 1974: 10). Si spiega, così, come l'io narrato si imbatta, nelle strade di Milano, in «zampilli e [...] cascate dell'oro» (VO, 156). Subito dopo, personificazioni, metafore, anafore, sinestesie sono segno della continua interazione fra letteratura e arti figurative, come avverrà nella fase del realismo magico, mentre la luce rappresenta la volontà di ricostruire il mondo:

Fuori, in quel frattempo, la Volontà di vivere aveva lanciato attorno manate di luce, che s'erano impiastrate contro gli sporti delle botteghe, s'erano appese ai cornicioni dei tetti; e di quella luce n'era sparsa in terra, sotto i piedi, nell'aria, dappertutto. La Volontà di vivere gridava dalle ruote delle carrozze e dalle campanelle dei tranvai. (VO, 156)

Sulla stessa linea si pongono le notazioni coloristiche, che, unendosi a suggestioni musicali, creano sinestesie, presenti pure quando l'io narrato immagina di far sorgere via Belloveso: ${ }^{12}$

S'èleva al mio cospetto la forma di un audace altare, e scintilla di molti colori; più bassa gli gira attorno un' ara di marmo a venature violacee con un vasto orlo d'arabeschi dorati; in alto ai due lati dell'altare quattro marmoree candele hanno per fiamme lampadine elettriche dall'acuta

\footnotetext{
${ }^{11}$ Come si ricorderà, Bontempelli, all'inizio dei Quattro preamboli, afferma: «Il còmpito più urgente e preciso del secolo ventesimo, sarà la ricostruzione del tempo e dello spazio. / Dopo averli ricostituiti nella loro eternità, nella loro immobilità, nella loro gelidezza, avremo cura di ricollocarli al posto che avevano perduto, nelle tre dimensioni infinite, fuori dell'uomo» (BONTEMPELLI, 1974: 9).

${ }^{12}$ «E così forte e solenne fu il mio grido interiore, che le poche ville pretenziose, sùbito intimorite, si scostarono ognuna dal suo luogo, e portandosi via le torrette rosse e i cancelletti di ferro battuto, s'allontanarono e scomparvero; e insieme i pali di ferro e gli alberi di legno dileguarono; poi dalla terra bigia cominciarono a scaturire fasce di biancori gelidi che rapidamente al mio sguardo impietrivano allineandosi in una duplice fuga parallela, accennavano per un istante l'ondulamento d'un ritmo di danza; poi si fermarono, elasticamente immobili e altissimi, guardando tutti a me con le pupille nere e rettangolari d'un numero infinito di finestre simmetriche: diciotto e diciotto eccelsi edifizi, in due file che andavano a incontrarsi e perdersi in direzione della lontana e invisibile malinconia delle regioni lacustri; diciotto e diciotto grattacieli di cemento armato; la mia creazione: via Belloveso» (VO, 205-206).
} 
punta. Anche, a tratti, questo altare suscita, a me che lo contemplo, la vaga memoria d'un organo, [...]. (VO, 208)

$\grave{E}$, ancora, secondo i principi del realismo magico che la dimensione terrestre viene superata: l'individuo che cercava via Belloveso «magicamente» scompare, «vaporando nell'etere», molto probabilmente «assunto, definitivamente o provvisoriamente, nei cieli» (VO, 198). Analogamente, per «prodigio» ogni piano di un edificio «si venne spostando qua e là orizzontalmente nell'aria [...] Poi il suolo riassorbì anche quella distesa di piani $[\ldots] »(\mathrm{VO}, 206)$. Un particolare tipo di specchio, lo «specchio allocatoptrotico» $(\mathrm{VO}, 259)$ è, poi, un «vero e proprio sintonizzatore di immagini diafane, eteree» (FABBRI, 2001) con cui il realismo magico consegue «i risultati migliori» (FABBRI, 2001). Contribuiscono a questo metafore e paragoni

Dalle radici alle cime mi squassò un brivido che parve squarciarmi (VO, 259);

lo specchio davanti a me s'era annebbiato come di veli grigi che tutto lo fluttuavano (VO, 260);

lo sguardo $[\ldots]$ ancora vagava come disperso in un diverso etere» $(\mathrm{VO}, 260)$;

mi disse come in un soffio $(\mathrm{VO}, 260)$;

gli occhi non si chinarono, neri e fondi come li avevo visti un vespero in riva al lago gemente» (VO, 260-261);

a quel moto il campo dello specchio come in un baleno si vuotò (VO, 261);

Lo specchio si scombuiò tutto di nuvole, come poco innanzi (VO, 261);

sinestesie

nella sùbita oscurità anche l'immane silenzio mi parve confortevole (VO, 259);

Sùbito il silenzio si tacque, s'animò di lontani sussurri diffusi, voce d'un'atmosfera [...] nuova [...] ebbi cuore di riaprire gli occhi $(\mathrm{VO}, 260)$;

Anche le sue labbra vidi agitarsi, e udii la voce che diceva. (VO, 260)

Il corpo umano appare, quindi, come un'entità a sé stante, secondo un procedimento abbastanza usato da Bontempelli: «in faccia a me era seduta una forma umana, era la forma di Laura» (VO, 260); «le nostre forme e le nostre voci eran vicine, sì che a un punto le anime crederono toccarsi» (VO, 261).

Si comprende, dunque, come ricorrano i termini «stupefazione» (VO, 195, 239), «stupefatto» (VO, 269), «stupefacente» (VO, 279), «stupore» (VO, 216, 271), «candore» (VO, 240). Questa parola, in particolare, sembra avere lo stesso significato che Bontempelli vi attribuirà nel discorso Pirandello o del candore:

Le anime candide [...] Sono quelle che per gran privilegio possiedono, di nascita, la naturalezza semplice che altre non raggiungeranno se non per una attenta elaborazione di tutta la vita. / La prima qualità delle anime candide è la incapacità di accettare i giudizi altrui e farli propri. [...] Il candore è al punto più lontano dalla ipocrisia; [...] Il candido, essendo all'opposto dell'ipocrita, 
è lontano quanto è possibile dal formalismo e dal cerimoniale, [...]. (BonTEMPELLI, 1978: 812813)

$\mathrm{Ne}$ La vita operosa, come si ricorderà, il termine 'candore' è riferito, a garantire il valore della scena di quotidianità presentata, ad un sacerdote, che, abituato a bere caffè senza zucchero, «gode del senso d'una particolare situazione di superiorità [...] ricava un gaudio raffinato e continuamente rinnovato del trovarsi a vivere nel periodo storico della saccarina» (VO, 240).

In una condizione simile perviene l'io narrato alla fine della vicenda. Quando esita ad alzarsi per recarsi al colloquio con il ministro, pensa: «Oh quanto sarebbe più nobile accontentarsi del piccolo bene presente: qualche ora di buon sonno» (VO, 284). Contemporaneamente, di nuovo attraverso il linguaggio del corpo («dei peli facciali», (VO, 284)), nella sua mente il ministro assume sembianze ridicole, mentre gli appare l'immagine della «donna che avevo vista passare impellicciata e profumata per le vie di Milano» (VO, 284), palese richiamo alla donna «bellissima» che, con una «corta e densa [...] pelliccia e lunghe e rade [...] calze» (VO, 151), l'io narrato incontra appena arriva a Milano: la città, perciò, influenza le sue azioni e il suo modo di pensare, come avviene ne La vita intensa. La rinuncia dell'io narrato all' appuntamento con il ministro rappresenta, quindi, la conclusione di un processo di formazione che gli fa preferire una vita semplice e genuina alle ambizioni materiali. Non a caso, dunque, l'ultima sezione si intitola, leopardianamente, Idillio. In Leopardi l' "uomo solo", difatti, Bontempelli sottolineerà come «l' "uomo solo" [...] in parte coincide con l'uomo candido, [...].» (1978: 836)

La conclusione, inoltre, consente di individuare nel testo valutato, insieme a La vita intensa, un antiromanzo, in quanto «Le due opere» smonterebbero «i meccanismi del romanzo ottocentesco» (FARINELLI, 2012: 30n), un primo livello, corrispondente ad un vero e proprio romanzo, la cui unitarietà sarebbe garantita dalla figura del protagonista, e in cui si potrebbero distinguere una situazione iniziale (l'io narrato è in guerra), il turbamento dell' equilibrio (ritorna a Milano), l'evoluzione della vicenda (le varie 'avventure'), lo spannung (è passato un mese dal ritorno a Milano dell'io narrato, che si sente travolto dal «turbine della metropoli» [VO, 247]), la conclusione. La presenza, all'interno del testo, di un' architettura, di una precisa organizzazione è testimoniata, del resto, dai nove capitoli, ognuno dei quali è diviso in sei sezioni. A volte vi sono dei rimandi fra una parte e l'altra: «Questo grido m'era sgorgato dall'anima per il ricordo dello sciocco séguito ch'ebbe la mia scarrozzata con uno sconosciuto, come si narra nel capitolo intitolato "Per Belloveso"» (VO, 217). Altre volte l'io narrante sottolinea, in modo ironico, l'autonomia delle varie sezioni: «Se tuttavia il lettore del presente capitolo non ha letto i sei che precedono, non importa: per l'intelligenza di questo basta ch'egli sappia che la mattina del 9 di febbraio, alle ore 10 e 45, mi venne il mal di capo» (VO, 248). Ciò è segno dell'oscillare di Bontempelli fra la consapevolezza della crisi dei tradizionali mezzi espressivi e la conseguente esigenza di sperimentare nuovi moduli, ovvero del contemperamento, tipico del modernismo, fra tradizione e innovazione. 
Il grande spazio concesso all'io narrante, difatti, contribuisce a determinare un secondo livello narrativo, in cui l'impalcatura del romanzo pare sgretolarsi.

\section{- L'io narrante}

L'io narrante mostra, sin dalle prime pagine del romanzo, di non svolgere, solo ed esclusivamente, una funzione narrativa. Dialoga con i narratari, presenta la città di Milano come luogo simbolo di quello che sarà il programma novecentista. Da una parte, infatti, la città è avvolta da un'atmosfera magica

Il cielo era coperto e l'aria un velo grigio; ma di tratto in tratto le vie s'illuminavano di lunghi bagliori folgoranti, perché rapide correnti d'oro invadevano il cielo, s'insinuavano tra le linee dei tetti, volavano sopra le strade della città con una voce d'areoplano giovane. Le correnti dell'oro a ogni momento urtando negli spigoli dei tetti si frangevano e mandavan giù rutilanti cascate a zampillar sui marciapiedi sotto lo sguardo dei passanti [VO, 152],

dall'altra ha dei contorni reali, sottolineati dagli spostamenti dell'io narrato, che ne consentono di tracciare una vera e propria pianta. ${ }^{13}$ Gli spazi, del resto, esprimono la nuova società, nata all'indomani della guerra: «La Volontà di vivere gridava dalle ruote delle carrozze e dalle campanelle dei tranvai» (VO, 156); «Anche nelle vie per le quali camminavo [...] giungeva l'eco degli omaggi popolari al dio Oggi» (VO, 159). Arrivato davanti alla casa di Manzoni, lo interroga «con rispetto», ma costui frustra, «con ironia candidamente perfida, ogni richiesta di chiarimento» (ROSA, 1982: 179). «Aspettando in Galleria» (VO, 166), l'io narrato si mette al corrente della situazione della contemporanea letteratura. I luoghi influenzano gli stati d'animo del protagonista:

Ma arrivato in piazza Cavour, alla disoccupazione si aggiunge la perplessità. Da una parte si sforzano di verdeggiare i Giardini, dall'altra [...] scampanella via Alessandro Manzoni. A sinistra troverei un po' d'alberi, dell'acqua, e certi uccelli come nei francobolli delle collezioni; [...] Ma a destra la vita [...]. $(\mathrm{VO}, 161)$

Nel capitolo quarto, Per Belloveso, mano mano che la carrozza si inoltra fra strade e piazze «meno affollate e meno illustri», all'io narrato «l'anima s'andava fasciando di lenta malinconia». Poi, quando «la luce» si rifà «nitida [...] ritrovando il sorriso di vie e piazze note recuperai gli spiriti» (VO, 196).

Significativi anche gli spazi interni. Il «sotterraneo fumoso» (VO, 177), il «minuscolo bar cristallino» (VO, 185), la «trattoria» (VO, 189), il Caffè Campari (209), il «bar del Commercio» (VO, 263) scandiscono vari momenti di una quotidianità fatta di esteriorità e, perciò, priva di valori. Nel bar del Commercio, difatti, il narratore distingue «tre o quattro giovanotti fatti tutti alla stessa maniera come fossero stati colati in serie da uno stampo» (VO, 265). Inoltre, se la «minuscola stanzina» (VO, 169), assegnata all'io narrato nella B.A.I.A., corrisponde alla mediocrità delle sue mansioni, il salotto «semimondano» (VO,

\footnotetext{
${ }^{13}$ Il Dàimone guida l'io narrato «dal Corso nella piazzetta Belgioioso» (VO, 158). Successivamente sono menzionati piazza Duomo - luogo simbolo di Milano -, via Visconti, la Galleria Vittorio Emanuele.
} 
181) di via Monte Napoleone sottolinea il contrasto fra apparenza e realtà. L'io narrato pensa inizialmente che il "pescecane" sia Malco, e non Valacarda. Il loro aspetto esteriore, dal momento che non vi è alcun approfondimento psicologico, glielo lascia credere:

[...] a me e al Dàimone Valacarda era già piaciuto. Di statura mite, baffi piccoli e neri, appariva uomo di spirito aperto e sottile. Lo sentimmo fraterno, là, dove avevamo il secreto tremore di trovarci estranei. L'altro, no. [...] Malco [...]. Riconobbi in lui a prima vista la formula estetica e morale del pescecane tipo, [...]. (VO, 180)

Pochi e di scarso pregio, a mostrare l'esiguo spazio concesso alle descrizioni, sono gli oggetti su cui il narratore fa convergere l'attenzione: il pianoforte, una «mensoletta d'ottone, fissa al muro, di quelle a parecchi intagli», priva di pipe, sebbene adibita a tenerle «sospese» (VO, 183), un' acquaforte che offre l'occasione per mettere in rilievo la superficialità dei nuovi tempi. Valacarda asserisce:

Non dica "non m'intendo di pittura" [...] Si procuri cinque o sei frasi, e se ne intenderà. Comincerà con l'adattarle un po' a caso: poi quelle cinque o sei ne germineranno spontaneamente altre nella sua abitudine, e lei si troverà un vocabolario. Quando avrà un vocabolario critico, necessariamente le verranno delle idee critiche. (VO, 182)

Le parole sono, di conseguenza, più importanti dei contenuti ed entrambi sono in continua trasformazione, ad indicare la mancanza di punti fermi:

Prima della guerra c'erano le parole "sensibilità", "dinamico", "musicale"; oggi invece le pietre basilari del vocabolario critico sono "costruito", "corposo", "architettura". Un vocabolario di questo genere può durare dai tre ai cinque anni. Anche per il contenuto è così. (VO, 182)

L'episodio dimostra, al contempo, come possano essere considerati dei veri e propri racconti le varie 'avventure' dell'io narrato, che, perciò, diventa quasi un narratore di secondo grado. Del resto, La vita operosa reca, come sottotitolo, Nuovi racconti d'avventure. Interessante, per esempio, il caso del quinto capitolo; nella sesta sezione, l'io narrante presenta «una donna anziana» (VO, 221), che racconta la vicenda sua e «di tre donne giovani», vittime degli ingranaggi perversi della società cittadina successiva alla guerra.

In alcuni momenti l'io narrante compie digressioni con cui esercita la sua funzione ideologica. Commenta la vicenda esprimendo le proprie opinioni su aspetti e questioni della società contemporanea. Scivola nella metanarrativa parlando di questioni letterarie:

Questo "ah" non fu un "ah" di quelli grassi, sdraiati, episcopali, che nei dialoghi della vita indicano soddisfatta conclusione e lasciano l'animo pacato: fu un "ah" arido, giallo di sarcasmi. I romanzieri non hanno ancora trovato la maniera di distinguerli nella scrittura, e mettono "ah" senz'altro, in tutt'e due i detti casi e anche nei loro infiniti intermedi e collaterali: la quale è una lacuna non lieve dell' arte nostra. (VO, 193)

Riflette sui comportamenti umani:

È universale il tremore del nuovo. Ognuno può riscontrarlo entrando in un caffè, in un salotto, in una biblioteca, in un bar automatico, in un tribunale civile o penale, in un qualunque pubblico o 
privato luogo, per la prima volta in vita sua. Tale tremore è fatto soprattutto di pudore. (VO, 214)

Ma l'uomo in istato di terrore rifugge dalle soluzioni più semplici del problema che lo assedia: e non l'uomo soltanto, ché vedemmo gli uccelletti dal ramo, sgomenti alla vista d'un cobra, precipitarsi giù tra le sue fauci invece di volar via come sarebbe loro assai facile. (VO, 216)

[...] s'è conservato modesto; non ha preso né moglie ricca né amanti costose, e neppure ha cambiato il sarto, né s'è messo a comprare romanzi con la copertina illustrata: le quali cose lo distinguono da altri arricchiti dell'ora presente. (VO, 210)

A volte il narratore cede la sua funzione ideologica a qualche personaggio. Il «pescecane» (VO, 188), per esempio, mette in rilievo, attraverso enumerazioni, $\mathrm{i}$ condizionamenti imposti allo scrittore dalle leggi del mercato:

Perché lei è uno scrittore e agli scrittori per farsi leggere occorrono figurazioni precise: il demonio, l'angelo, il pescecane grasso e cùpido, il fante energico e macerato. [...] Loro scrittori debbono essere o sentimentali o cinici, se no il pubblico si disorienta. (VO, 188)

Questi commenti e le digressioni avvicinano il nostro testo al saggio.

\section{Conclusione}

Le diverse funzioni dell'io che abbiamo cercato di distinguere si intersecano continuamente, quasi come avviene nell'autofiction, considerata, secondo la definizione di Donnarumma,

una narrazione in cui, come in un' autobiografia, autore, narratore e protagonista coincidono; ma in cui, come in un romanzo, il protagonista compie atti che l'autore non ha compiuto, e ai fatti riconosciuti come empiricamente accaduti si mescolano eventi riconoscibili come non accaduti. (DONNARUMMA, 2014: 130)

In tal modo, Bontempelli arriva ad una disarticolazione dell'intreccio.

Egli sviluppa, del resto, un procedimento già presentato ne La vita intensa, in cui autore, narratore e personaggio si intrecciano sin dalla prima pagina. «Racconto fatti veri, accaduti a me, nella città di Milano. [...] questa storia è vera così. E io non la scrivo per quegli uomini troppo complicati» ${ }^{14}$ (VI, 7). Il secondo romanzo, poi, inizia con una Lettera dedicatoria alla signorina Ardita firmata dall'Autore. È ancora costui a sostenere la necessità di scrivere «un capitolo di pura teoria. Il quale potrà servire a ogni lettore, sia per l'intelligenza del romanzo stesso, sia in molti e varii casi della vita» (VI, 17). Quindi cede la parola all'io narrante: «questo è il fondamento filosofico di tutta l'avventura che sto per narrarvi, la quale ebbe luogo in Milano il giorno 10 di marzo di un certo anno» (VI, 18). Tale precisazione cronologica, il fatto che l'io narrato si chiami Massimo e suo figlio Mino, il riferimento alle sue svariate occupazioni, l'allusione alla rivista «La Vraie Italie», diretta da Papini, alla rivista «Ardita» (VI, 30) il rilievo dato ai ricordi («Non ricordavo, certo, più con esattezza dove mi trovavo. [...]

${ }^{14}$ Le citazioni dalla Vita intensa, indicata con la sigla VI, si riferiscono a BonTEMPELLI, 1978. 
Non ricordando altro, $[\ldots] »(\mathrm{VI}, 22)$; «Ricordo che una volta un gaio e grasso avventore aveva scherzato su quel cartello» (VI, 56)), il contatto costante con i narratari («Io non so se il lettore ricorda che io ero senza sigarette» (VI, 20); «Poiché il lettore non è giovane com'ero io allora, ha già capito che il giorno appresso nessun ciclo rotò a soffermarsi davanti alla mia porta» (VI, 67)), insieme ad altre forme della metalessi dell'autore («Questo passo indietro lo farete in compagnia modesta e piacevole, cioè con me» (VI, 50)), potrebbero far pensare ad una autobiografia. Se, poi, vari dettagli relativi al tempo e allo spazio

Mi trovai in piazza della Scala, al bar annesso alla farmacia» (VI, 39);

«Tutti i martedì esco di buon mattino e comincio a fare il giro dei tabaccai eccentrici» (VI, 50);

«Il $31 \mathrm{mi}$ svegliai con la commozione che in quella giornata avrei preso il primo danaro guadagnato nella mia vita» (VI, 63);

«Davo ripetizione di pianoforte in via dei Serpenti, numero $x$, piano secondo» (VI, 80)

dovrebbero rendere il più possibile verosimili le diverse vicende, varie situazioni e personaggi sono palesemente infondati: il quarto romanzo si svolge il 31 aprile, nel sesto romanzo appare il demone, che sottolinea, ancora, l'intreccio fra io autore ed io personaggio. Leggiamo, infatti:

Il mio demone, forse quello stesso demone che oggi mi suggerisce finti romanzi allo scopo di pigliare in giro i lettori di Ardita, allora mi suggerì un romanzo vivo per risolvere le ragioni materiali della mia esistenza; e mi mostrò a me stesso, in seguito a una rapida e romanzesca avventura d'amore, sposo borghesemente felice di quel biondo e di quell'azzurro, che dovevano essere figli o sorelli [sic] delle ricchezze accumulate in quel terzo piano. (VI, 80)

L'io narrante racconta poi di essersi suicidato, di essere stato «sepolto senza onori» (VI, 91). Alcuni anni più tardi, però, si sposa e comincia «a fare il romanziere» (VI, 91). Ed ugualmente privo di base reale è l'incontro dell'io autore con i suoi personaggi nel Romanzo dei romanzi. Tutto ciò sembrerebbe autorizzare il richiamo ad alcuni aspetti dell'autofiction. A questo proposito, possiamo notare come, secondo Vita Giordano, la domanda dell'io narrante («Esiste davvero una signora Marta Calabieri, trentacinquenne e gelosa, nel mondo? $\mathrm{O}$ me la sono figurata io?» (VI, 133)) ricorderebbe «quella che verrà scritta in uno dei più noti racconti di metafiction, "Lost in the Funhouse" (1968) di John Barth ("Is there really such a person as Ambrose, or is a figment of the author's imagination?")» (GIORDANO, 2009: 40). L'io narrante, cioè, che ha esordito sostenendo inizialmente di raccontare «fatti veri, accaduti a me, nella città di Milano» (VI, 7), si diverte a disorientare il lettore, a manipolare l'io personaggio.

Questo accade anche ne La vita operosa grazie al linguaggio ironico. E, a proposito delle conseguenze che esso esercita sull'io, Saccone si rifà a quanto affermato da Paul De Man, sulla scia della «nozione baudelairiana [...] del dédoublement come modalità costitutiva essenziale del comique absolu»:

"Il linguaggio ironico taglia il soggetto in un io empirico che vive in uno stato di inautenticità e in un altro io, che esiste solo nella forma di un linguaggio che afferma, che dichiara la 
conoscenza di quest'inautenticità" - e aggiunge significativamente per noi: "Questo, ovviamente, non trasforma il suo linguaggio in un linguaggio autentico: perché la conoscenza dell'inautenticità non è la stessa cosa dell'essere autentico" (SACCONE, 1979: 26).

È evidente, dunque, l'importanza de La vita operosa nell'ambito della produzione di Bontempelli, il cui progetto di rinnovamento letterario non si deve solo identificare con il realismo magico.

\section{BIBLIOGRAFIA}

BALDACCI Luigi (1959), Massimo Bontempelli, in Belfagor, 14, p.432-446.

BONTEMPELLI Massimo (1974), L'avventura novecentista, Firenze, Vallecchi.

BontemPelli Massimo (1978), BALDACCi Luigi (éd.), Opere scelte, Milano, Mondadori.

Donnarumma Raffaele (2006), Gadda modernista, Pisa, ETS.

DONNARUMMA Raffaele (2014), Ipermodernità. Dove va la narrativa contemporanea, Bologna, Il Mulino.

FABBRI Fabriano (2001), Bontempelli e Malaparte: le due vie genetiche per il '900, in : «Bollettino '900», n.1, giugno www.boll900.it/numeri/2001-1/Wbol/Fabbri/Ffabbri_frame.html

FARINELLI Patrizia (2012), Oltre il tempo attraverso il mito: Bontempelli e l'attualizzazione narrativa di un progetto teorico - da Eva ultima al Viaggio d'Europa, in : Ars \& Humanitas / Študije, L.6, 2, p.23-35.

FARINELLI Patrizia (2016), L'isola del novecentismo nell'arcipelago modernista, in BALDASSARRI Guido, Di IASIO Valeria, FERRONI Giovanni, PIETROBON Ester (éd.) I cantieri dell'italianistica. Ricerca, didattica e organizzazione agli inizi del XXI secolo. Atti del XVIII congresso dell'ADI (10-13 settembre 2014), Roma, Adi editore. http://www.italianisti.it/Atti-di-Congresso.

Fontanella Luigi (1997), Storia di Bontempelli. Tra i sofismi della ragione e le irruzioni dell'immaginazione, Ravenna, Longo.

GALATERIA Marinella Mascia (1977), Tecnica della sorpresa e romanzo comico di Massimo Bontempelli. Saggio su La vita intensa e La vita operosa, Roma, Bulzoni.

GIORDANO Vita (2009), Dalle avventure ai Miracoli. Massimo Bontempelli fra narrativa e metanarrativa, Leicester, Troubador Publishing Ltd.

LUPERINI Romano (2012), Il modernismo italiano esiste, in : LUPERINI Romano ; TORTORA Massimiliano (éd.), Sul modernismo italiano, Napoli, Liguori, p.312.

PIRANDELlo Luigi (1937), Novelle per un anno, Milano, Mondadori, I.

RosA Giovanna (1982), Il mito della capitale morale. Letteratura e pubblicistica a Milano fra Otto e Novecento, Milano, Edizioni di Comunità.

SACCONE Antonio (1979), Massimo Bontempelli. Il mito del '900, Napoli, Liguori. 